\title{
Diagnostic Accuracy of Skin Cancer by Family Physicians
}

\author{
Sarina Trejić, MD, Hans J. G. Peters, Satish F. K. Lubeek, MD, PhD, and \\ Floris A. van de Laar, MD, PhD
}

Background: Skin cancer is the most common type of cancer worldwide. Family physicians (FPs) need to differentiate between nonmalignant and malignant skin conditions, but the diagnostic accuracy of FPs has never been studied in primary care.

Aim: To assess the accuracy of skin cancer diagnoses by FPs. Our secondary aim was to analyze the number of patients with premalignant lesions and examine the diversity of skin-related questions in Dutch primary care.

Method: This study is a retrospective cohort of all new skin-related health questions between January 1 , 2018, and July 1, 2018, in a Dutch primary care registration network with data from 26 FPs in 6 practices, with a follow-up of at least 1 year. The initial FP diagnosis was dichotomized as malignant or nonmalignant and compared in a crosstab to the final diagnosis registered after the follow-up period (reference standard).

Results: Our study population included 2952 patients. During the research period, 35 patients received a final diagnosis of skin cancer. The sensitivity and specificity of the FP diagnosis of malignancy was $74.3 \%$ (95\% confidence interval [CI], $56.7 \%$ to $87.5 \%$ ) and $97.3 \%$ (95\% CI, 96.7\% to 97.8\%), the positive predictive value and negative predictive value was $21.5 \%(95 \% \mathrm{CI}, 17.2 \%$ to $26.5 \%)$ and $\mathbf{9 9 . 7 \%}$ (95\% CI, $99.5 \%$ to $99.8 \%$ ), respectively. Seventy-two patients were diagnosed with a premalignant lesion. Included patients received 141 different diagnoses.

Conclusion: The calculated diagnostic accuracy of FPs is high and shows that FPs are especially accurate in excluding malignancy. This research shows the variety of skin problems in primary care and shows that the FP can deliver safe and effective dermatologic care. ( $\mathrm{J}$ Am Board Fam Med 2021;34:984-990.)

Keywords: Family Physicians, Primary Health Care, Retrospective Studies, Sensitivity and Specificity, Skin Cancer

\section{Introduction}

Skin-related problems are an important part of the workload in primary care. Nearly $14 \%$ of all presented help requests are skin-related. ${ }^{1}$

Skin cancer is the most common type of cancer worldwide, making up $52 \%$ of all new cancer

This article was externally peer reviewed.

Submitted 23 February 2021; revised 31 May 2021; accepted 4 June 2021.

From the Department of Primary and Community Care, Radboud University Medical Centre, Nijmegen, The Netherlands (ST, HJGP, FAVDL); Department of Dermatology, Radboud University Medical Centre, Nijmegen, The Netherlands (SFKL).

Funding: None.

Conflict of interest: None.

Corresponding author: Dr. F. A. van de Laar, Department of Primary and Community Care, Radboud University Medical Centre, Geert Grooteplein 21, 6525 EZ Nijmegen, The Netherlands, Telephone: +31-24 36181 81, Fax: +31 -243635120 (E-mail: Floris.vandeLaar@radboudumc.nl). diagnoses in the Netherlands. ${ }^{2,3}$ In 2018, the overall incidence of skin cancer in primary care was 3.2 per 1000 person-years. The incidence of melanoma was 0.4 per 1000 person-years, basal cell carcinoma (BCC) was approximately 2.3 per 1000 person-years, and the incidence of squamous cell carcinoma (SCC) was approximately 0.9 per 1000 person-years. ${ }^{4-6}$

Timely differentiation between malignant and nonmalignant skin conditions enhances early-stage treatment and might thereby improve prognosis. ${ }^{7,8}$ However, the spectrum of skin cancer and premalignant skin lesions is broad, and in early stages, recognition may be particularly difficult.

Previous research on the diagnostic accuracy of skin cancer by family physicians (FP) was performed in selected populations. In a systematic review by Chen et $\mathrm{al}^{9}$ concerning the accuracy of the FP diagnosis in melanoma, the presented outcomes were based on, for example, diagnosing 
pictures of skin lesions. Swetter et $\mathrm{al}^{10}$ calculated a diagnostic accuracy based on the screening of healthy veterans. Marra et $\mathrm{al}^{11}$ studied referral letters with a written FP diagnosis. Ahmadi et al ${ }^{12}$ calculated the diagnostic accuracy based on patients with a lesion suspected to be malignant by the FP in the Netherlands. The consequence of researching in a selected patient group is that the diagnostic accuracy in the general primary care population remains unknown. Therefore, our primary aim was to calculate the diagnostic accuracy of general practitioner's diagnosis concerning skin cancer in the Dutch adult primary care population.

Our secondary aim was to gain insight into the diagnostic process of patients with skin questions. We aimed to analyze the number of patients with premalignant lesions and examine the diversity of skin-related health questions in primary care.

\section{Materials and Methods Study Design}

This study was a retrospective cohort study with data from the practice-based Family Medicine Network (FaMe-net) of The Department of Primary and Community Care (ELG) at the Radboud University Medical Centre in Nijmegen. FaMe-net collects data from 26 individual FPs working in 6 general practices in different regions of the Netherlands.

FaMe-net systematically and prospectively registers data on episodes of care for all patients registered to the practice. An episode is defined as an individual health problem that includes all information and actions from the first to the last consultation related to this specific problem. The title of an episode represents the most probable diagnosis at a certain time point and may thus change over time when a condition evolves, and more (diagnostic) information (eg, from specialist consultation) becomes available. Diagnoses are coded with the International Classification of Primary Care, Second Edition (ICPC-2) and International Classification of Diseases, Tenth Revision (ICD-10) classification systems to code procedures and diagnosis. ${ }^{13}$ Further, the reasons for encounter (RFE) and all interventions such as excisions and referrals within an episode are registered and coded. The RFE reflects the initial presentation of the problem by the patient to the doctor, for example, a symptom (I noticed a spot), a (self-) diagnosis (I have a nevus), or a request for intervention (I want an excision). An RFE may also be doctor-driven when a doctor initiates an episode, for example, when the FP notices a suspect nevus during a routine examination. Participating FPs meet regularly to discuss registration issues and improve the quality of registration. More details about FaMe-net are published in this journal recently. ${ }^{14}$

In the Netherlands, patients are registered to 1 practice, and all encounters with other care providers in secondary and tertiary care are reported back to the FP. This type of encounter enables an integral and thorough overview of all health care encounters of each patient.

\section{Study Population}

All adults who consulted the FP between January 1, 2018, and July 1, 2018, with a skin-related RFE (ICPC-2 codes starting with S), were included. The definition of RFE is the health question formulated by the patient. The RFE is mostly patient driven but can be initiated by the FP, for example, when a FP signals a lesion by coincidence. How long this health question has been present at the first presentation is called the RFE duration. FPs must register both the RFE and the RFE duration to proceed with the administration of the consultation. All patients were followed up for at least 1 year after the study period to assess whether skin cancer was diagnosed. Patients who moved away or changed FP before that time were excluded. Patients who passed away or moved to a nursing home before the follow-up period of a year remained included.

\section{Definitions}

We dichotomized the FPs diagnoses into 'malignant' and 'nonmalignant' as follows. A diagnosis was categorized as malignant when the FP (a) documented a malignant diagnosis in the encounter, (b) referred to the dermatologist with the specific request for further diagnostics/therapy on skin cancer, or (c) performed a punch or excision biopsy with pathologic analysis of the tissue. In all other cases, the diagnosis was categorized as nonmalignant. FPs often have multiple encounters with a patient before making a diagnosis. The acceptable period for making a diagnosis as defined by registering FPs and current guidelines was: 10 weeks for BCC, 5 weeks for SCC, and 2 weeks for melanoma. ${ }^{15}$ If FPs suspected malignancy after these 
Figure 1. Flowchart of study population. Abbreviation: RFE, reasons for encounter.
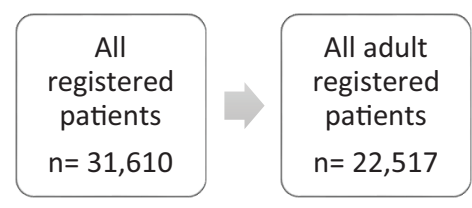

timeframes, we considered the FP diagnosis as nonmalignant.

The reference standard was defined as the final diagnosis registered after the follow-up period of at least 1 year, based on the assumption that symptomatic clinical progression of skin cancer occurs within a year.

Further stratification of skin cancer was performed using 4 categories: melanoma, BCC, SCC, and other (rare forms of skin cancer). ${ }^{5}$ Examples of premalignant skin conditions include actinic keratosis (AK), SCC in situ (or Bowen's disease), and dysplastic nevus.

\section{Data Extraction}

Data concerning patients with a skin question were extracted from FaMe-net. Data regarding all patients with a final diagnosis of skin cancer in FaMe-net were compared with the patients in the study population to verify that all patients with skin cancer contacted the FP with a skin-related RFE.

We combined data from the electronic database with file research to elicit data about the FPs diagnoses. We researched the files of all patients: (a) who were referred, (b) where tissue was pathologically analyzed, (c) with a malignant diagnosis in the episode title. In addition, we performed file

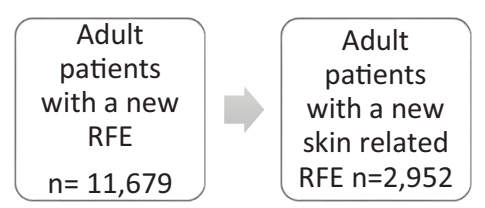

research to correct coding errors, such as double coding of the same episode.

\section{Analysis}

Statistical analysis was performed with Statistical Package for Social Sciences (SPSS) for Windows, version 25 . Only descriptive statistics were used. To calculate the confidence interval, MedCalc's Diagnostic test calculator was used. ${ }^{16}$

First, we analyzed the episodes of skin cancer. A crosstab was generated to calculate the diagnostic accuracy of the FP diagnosis of skin cancer. Next, the premalignant skin conditions were studied. Lastly, we analyzed the RFEs and all other diagnoses.

\section{Results}

\section{Incidence and Characteristics}

The total number of registered patients in the FaMe-net practices at the start of the research period was 31,610; of this group, 22,517 patients were adults. The age differentiation of the registered patients was similar to the general Dutch population. ${ }^{17}$ In the research period, 11,679 adult patients consulted the FP with 1 or more new health questions, resulting in 22,861 new episodes. Of this

Table 1. Patient Characteristics

\begin{tabular}{|c|c|c|c|}
\hline & All Registered Patients n (\%) & Adult Patients New RFE n (\%) & Adult Patients Skin RFE n (\%) \\
\hline Total & 31,610 & 11,679 & 2,952 \\
\hline \multicolumn{4}{|l|}{ Sex } \\
\hline Male & $15,982(50.6)$ & $4762(40.8)$ & $1,248(42.3)$ \\
\hline Female & $15628(49.4)$ & $6917(59.2)$ & $1,704(57.7)$ \\
\hline \multicolumn{4}{|l|}{ Age, years } \\
\hline$\leq 17$ & $9,093(28.8)$ & - & - \\
\hline $18-29$ & $4,423(14.0)$ & $1,883(16.1)$ & $473(16.0)$ \\
\hline $30-49$ & $9,165(29.0)$ & $4,541(38.9)$ & $1,128(38.2)$ \\
\hline $50-69$ & $6,517(20.6)$ & $3,490(29.9)$ & $879(29.8)$ \\
\hline$\geq 70$ & $2,412(7.6)$ & $1,765(15.1)$ & $472(16.0)$ \\
\hline
\end{tabular}

RFE, reason for encounters. 
Table 2. Crosstab to Calculate Diagnostic Accuracy of the Family Physician Diagnosis

\begin{tabular}{lccc}
\hline & $\begin{array}{c}\text { Final Diagnosis } \\
\text { Malignant }\end{array}$ & $\begin{array}{c}\text { Final Diagnosis } \\
\text { Nonmalignant }\end{array}$ & Total \\
\hline $\begin{array}{c}\text { FP diagnosis } \\
\text { malignant }\end{array}$ & 26 & 95 & 121 \\
$\begin{array}{c}\text { FP diagnosis } \\
\text { nonmalignant }\end{array}$ & 9 & 3,362 & 3,371 \\
Total & 35 & 3,457 & 3,492 \\
\hline
\end{tabular}

FP, family physician.

group, 2,952 patients consulted the FP with 1 or more new skin-related health questions, resulting in 3,492 episodes. This shows that $15.3 \%$ of all new health questions in adults in the general practice are skin-related.

Figure 1 shows a flowchart of the included patients. Table 1 shows the baseline characteristics of the patient groups.

\section{Skin Cancer}

In the research period, 35 patients received a final diagnosis of skin cancer. Of these patients, 27 were diagnosed with BCC, 5 with SCC, and 3 with melanoma. All patients with skin cancer consulted the FP with a skin-related RFE, so all patients were part of our research group. The incidence of skin cancer among all registered adults $(\mathrm{n}=22,517)$ was $0.2 \%$ in 6 months. In patients with a skin-related RFE $(n=2952)$, the incidence was $1.2 \%$ in 6 months. In Table 2, the crosstab that was used to calculate the diagnostic accuracy is shown.

The sensitivity of the FP diagnosis concerning skin cancer was $74.3 \%$ (95\% confidence interval [CI], $56.7 \%$ to $87.5 \%$ ) and the specificity $97.3 \%$ (95\% CI, $96.7 \%$ to $97.8 \%$ ). The PPV was $21.5 \%$ (95\% CI, $17.2 \%$ to $26.5 \%$ ) and the NPV $99.7 \%$ (95\% CI, $99.5 \%$ to $99.8 \%$ ).

In 9 cases, the FP suspected a nonmalignant condition, but the final diagnosis was malignant (6 BCCs, 2 SCCs, and 1 melanoma). In 3 of these BCC cases, malignancy was suspected by the FP after 5,4 , and 3 months. In the other 3 BCC cases and the 2 SCC cases, the patients were referred to the dermatologist, but not with the explicit request to exclude skin cancer. In the melanoma case, the FP suspected melanoma after 3 weeks (1 week after the pre-set limit of 2 weeks). All these patients with an initial (false) nonmalignant diagnosis were eligible for curative treatment.

The cases where the FP suspected a malignant lesion, but the final diagnosis was nonmalignant $(\mathrm{n}=95)$, the most common final diagnoses were;

Figure 2. Reasons for encounters (RFE) from patients with skin cancer.

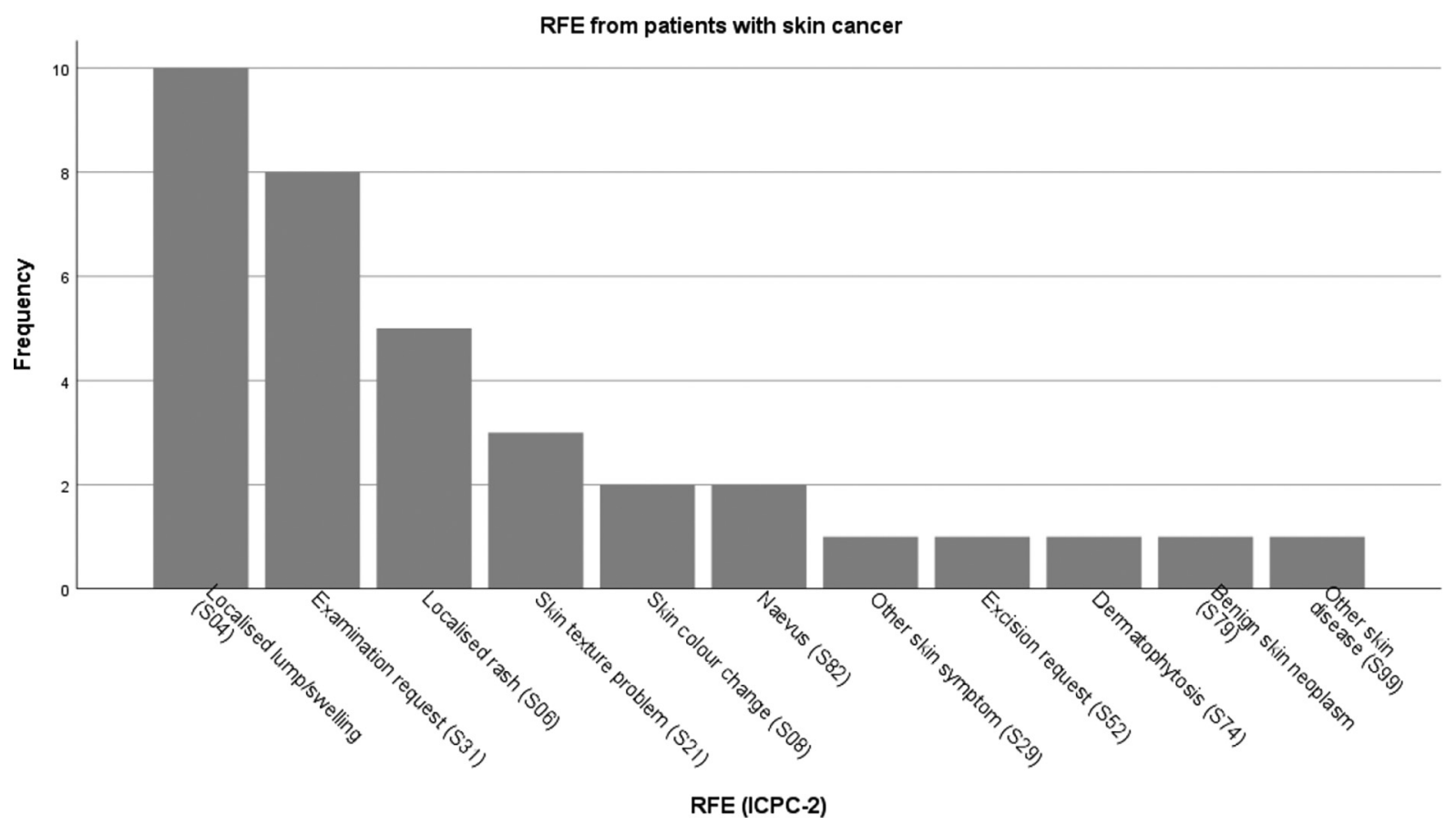


Table 3. Five Most Common Skin Reason for Encounters with Duration* and Interventions

\begin{tabular}{|c|c|c|c|c|c|c|}
\hline & $\begin{array}{c}\text { Number of } \\
\text { Episodes } \mathrm{n}(\%)\end{array}$ & $\begin{array}{c}\text { Median RFE } \\
\text { Duration }^{\dagger}\end{array}$ & $\begin{array}{l}\text { Range RFE } \\
\text { Duration }\end{array}$ & $\begin{array}{c}\text { Patients } \\
\text { Referred n (\%) }\end{array}$ & $\begin{array}{c}\text { Medication } \\
\text { Prescribed n (\%) }\end{array}$ & $\begin{array}{c}\text { Excisions/Remove } \\
\text { Biopsy/ } \\
\text { Destruction/ } \\
\text { Debride n (\%) }\end{array}$ \\
\hline $\begin{array}{l}\text { Rash localized } \\
\text { S06 }\end{array}$ & $484(13.0)$ & 2.0 & 1 hour-20 years & $35(7.2)$ & $259(53.5)$ & $22(4.5)$ \\
\hline $\begin{array}{l}\text { Lump/swelling } \\
\text { localized S04 }\end{array}$ & $472(12.7)$ & 8.6 & 1 hour - 35 years & $34(7.2)$ & $28(5.9)$ & $121(25.6)$ \\
\hline Pruritus S02 & $196(5.3)$ & 2.0 & 4 hours -5 years & $7(3.6)$ & $126(64.3)$ & $1(0.5)$ \\
\hline Nevus/mole S82 & $180(4.8)$ & 64.6 & 2 days -48 years & $21(11.7)$ & $3(1.7)$ & $44(24.4)$ \\
\hline $\begin{array}{l}\text { Laceration/cut } \\
\text { S18 }\end{array}$ & $137(3.7)$ & 2.0 hours & 1 hour-12.9 weeks & $3(0.7)$ & $31(22.6)$ & $9(6.6)$ \\
\hline
\end{tabular}

RFE, reason for encounters.

${ }^{*} \mathrm{RFE}$ duration is the time a health problem has been present at the time of the first family physician consultation.

${ }^{\dagger}$ Median RFE duration is presented in weeks.

melanocytic nevus (36.5\%), seborrheic keratosis $(17.3 \%)$, and actinic keratosis $(8.7 \%)$.

Most skin cancer patients were referred to the dermatologist (88.6\%). In 1 in 3 patients with skin cancer (31.4\%, 1 SCC, and 10 BCCs), a biopsy or excision was performed. Some patients were referred after a biopsy or excision (20\%). The average number of FP consultations in patients with skin cancer was 3.2. Figure 2 shows the RFEs from patients with skin cancer. The RFE is the health question from the patient, described in their words.

\section{Premalignant Skin Lesions}

In the study population, the final diagnosis in 72 patients was a premalignant skin lesion; this equals an incidence of $0.3 \%$ in all registered adults and $2.4 \%$ in patients with a skin-related RFE. Most patients with a premalignant lesion were diagnosed with $\mathrm{AK}(93 \%)$, others with Bowen's disease(2.8\%), melanoma in situ (2.8\%). Almost half (48.6\%) of all premalignant skin lesions were removed (excision or biopsy) by the FP. One in $5(20.8 \%)$ patients with a premalignant skin lesion were referred. Medication was prescribed to $8.3 \%$ of the patients with a premalignant skin lesion. On average, a patient with a premalignant skin lesion consulted the FP 2 times.

\section{other Skin-related Conditions}

The 5 most common RFEs with additional information about the RFE duration and interventions are shown in Table 3 . The most common diagnosis in patients with a 'localized rash' RFE and patients with a 'pruritus' RFE was contact/allergic dermatitis $(20.5 \%$ and $12.8 \%$, respectively). Patients with a 'localized lump/swelling' RFE were mostly diagnosed with a sebaceous cyst (13.1\%). The final diagnosis was identical to the RFE in patients with a 'nevus' RFE and patients with a 'laceration/cut' $\operatorname{RFE}(72.8 \%$ and $91.2 \%$, respectively).

Table 4. Five Most Common Diagnoses with Reason for Encounters Duration and Interventions

\begin{tabular}{|c|c|c|c|c|c|c|}
\hline & $\begin{array}{c}\text { Number of } \\
\text { Episodes n (\%) }\end{array}$ & $\begin{array}{l}\text { Median RFE } \\
\text { Duration }\end{array}$ & $\begin{array}{l}\text { Range RFE } \\
\text { Duration }\end{array}$ & $\begin{array}{c}\text { Patients } \\
\text { Referred n (\%) }\end{array}$ & $\begin{array}{c}\text { Medication } \\
\text { Prescribed n (\%) }\end{array}$ & $\begin{array}{c}\text { Excisions/ } \\
\text { RemoveBiopsy/ } \\
\text { Destruction/ } \\
\text { Debride n (\%) }\end{array}$ \\
\hline Dermatophytosis & $254(7.3)$ & 4.3 weeks & 1 day- -15 years & $3(1.2)$ & $21(8.3)$ & $3(1.2)$ \\
\hline $\begin{array}{l}\text { Contact/allergic } \\
\text { dermatitis }\end{array}$ & $226(6.5)$ & 3 weeks & 1 day- 20 years & $13(0.6)$ & $20(8.8)$ & $0(0)$ \\
\hline Nevus & $214(6.1)$ & 1 year & 2 days -48 years & $32(15.0)$ & $2(0.9)$ & $40(18.7)$ \\
\hline Laceration/cut & $151(4.3)$ & 2 hours & 1 hour-90 days & $5(3.3)$ & $3(2.0)$ & $9(6.0)$ \\
\hline Insect bite/sting & $149(4.3)$ & 2 days & 1 hour-30 days & $1(0.7)$ & $22(14.8)$ & $6(4.0)$ \\
\hline
\end{tabular}

RFE, reason for encounters. 
The 5 most common final diagnoses with RFE duration and interventions can be seen in Table 4 . The most common RFE was the same as the final diagnosis in patients with "dermatophytosis" (33.9\%), "nevus" (55.1\%), "laceration/cut" (77.2\%), and "insect bite/sting" (66.9\%). The most common RFE in patients with contact/allergic dermatitis was "localized rash" (40.9\%).

The incidences of some common skin problems as diagnosed by the FP were: $1.1 \%$ for dermatophytosis, $1.0 \%$ for contact/allergic dermatitis, $1.0 \%$ for nevus, $0.6 \%$ for seborrheic keratosis, $0.6 \%$ for warts, $0.5 \%$ for fibroma, $0.4 \%$ for sebaceous cyst, $0.2 \%$ for impetigo, $0.2 \%$ for herpes zoster, $0.1 \%$ for rosacea, $0.05 \%$ for acne and $0.05 \%$ for psoriasis.

All patients with skin-related health questions received in total 141 different final diagnoses. The range of diagnoses is broad, from common diagnoses such as described above to less common diagnoses as; domestic violence, auto-mutilation, and goiter.

\section{Discussion}

\section{Summary}

Our study found that $15.3 \%$ of all new health requests by adults in primary care were skin-related. We calculated the diagnostic accuracy in the patients with a skin-related RFE. The sensitivity was $74.3 \%$ (95\% CI, $56.7 \%$ to $87.5 \%$ ), the specificity was $97.3 \%$ (95\% CI, $96.7 \%$ to $97.8 \%$ ), the positive predictive value was $21.5 \%$ (95\% CI, $17.2 \%$ to $26.5 \%)$, and the negative predictive value was 99.7\% (95\% CI, $99.5 \%$ to $99.8 \%$ ). This finding suggests that FPs can detect more than 1 in 5 skin cancers despite the low incidence. In our research period of 6 months, 141 different diagnoses were made after a skin-related RFE.

\section{Strengths and Weaknesses}

The strengths of this research are that our study is the first to calculate the diagnostic accuracy of FPs diagnosing skin cancer in an unselected group of patients in primary care. The data used from $\mathrm{FaMe}-\mathrm{net}$ reflects reality as well as possible, providing information that was collected in real FP consultations by the FP. File research also enabled us to correct coding errors. The access to referral letters and consultation notes gave a broader look in interpreting the diagnostic process of the FP. Our definition of the FP diagnosis was based on consultation notes and the actions conducted by the FP and is therefore strong and reflects daily practice.
The reference standard in this research is good; it is unlikely that we missed cases of skin cancer in our research period. This study is the first to categorize patients by RFE, which gave insight into patients' interpretation and presentation of symptoms.

A weakness of this research is the low incidence of malignant skin lesions $(\mathrm{n}=35)$. We found only a few melanomas $(n=3)$, leading to the fact that it was not possible to draw a conclusion considering the diagnostic process of FPs exclusively in melanomas. Of all skin cancers, early recognition is most important for melanoma since this is the most dangerous type of skin cancer. Another weakness is that FPs diagnose more skin problems than they report. For example, during routine physical examination, FPs may see a lesion and directly recognize this as nonmalignant. These observations are not always registered because it was not the initial health question of the patient. This finding could mean an underestimation of our specificity and NPV. The skin color in our study group was not registered, but we suspect our study group was mostly fair-skinned. The diagnostic accuracy may therefore be different in a population that is not mostly fair-skinned.

\section{Comparison with Existing Literature}

We cannot compare our calculation of the proportion of skin-related questions based on RFEs to calculations in previous research because the existing literature ${ }^{1}$ studied the end diagnosis, which in many cases is different from the RFE.

The diagnostic accuracy we calculated is different from the numbers in the available research. ${ }^{9,10,18}$ The reason for this difference is a different case mix. In populations with different disease prevalence, the sensitivity, and specificity of test changes. ${ }^{19,20}$ Our research is the first to calculate diagnostic accuracy in a population with a low incidence. This means our diagnostic accuracy reflects the daily practice from the FP perspective more than the previous research.

Other research concerning skin diagnoses in primary care used a different coding system that only describes diagnoses, unlike today, where RFEs are coded separately from diagnoses. ${ }^{21}$ Our research is the first that was able to study different skin-related help requests.

\section{Implications for Research and Practice}

Our results show a diagnostic accuracy based on practice-based data. We demonstrated that this method is a good and reliable method for calculating 
the diagnostic accuracy in skin cancer. This method is a new way of doing research and could be used to calculate the diagnostic accuracy in more (skin) diseases.

Our results show high diagnostic accuracy in diagnosing skin cancer in primary care. This shows that the FP can deliver safe and effective dermatologic care, especially considering our strict definition of the FP diagnosis. The diagnostic accuracy might improve by appointing $1 \mathrm{FP}$ per practice who is more educated in dermatology to maintain the quality of diagnosis. The FP can provide proper care for many nonmalignant and even malignant lesions, therefore, providing good and affordable care for the community.

To see this article online, please go to: bttp://jabfm.org/content/ 34/5/984.full.

\section{References}

1. Vries E. Skin conditions at the FP and dermatologist. Available from: https://www.ntvg.nl/academie/ infographics/huidaandoeningen-bij-huisarts-endermatoloog. Published 2013. Accessed August 28, 2019.

2. Netherlands Comprehensive Cancer Organisation. Available from: https://www.iknl.nl/kankersoorten/ huidkanker. Published 2019. Accessed May 25, 2020.

3. Netherlands Comprehensive Cancer Organisation. Skin cancer in the Netherlands; figures from 30 years of Dutch cancer registration. Available from: https://iknlsawebprod.blob.core.windows.net/ mediacontainer/iknl/media/pdfs/kankersoorten/ iknl_huidkanker-in-nl_rapport_nkr.pdf. Published 2019. Accessed May 25, 2020.

4. Nivel care registration primary care. Incidence and prevalence. Available from: https://www.nivel.nl/nl/ nivel-zorgregistraties-eerste-lijn/incidenties-enprevalenties. Published 2019. Accessed November 28, 2019.

5. Dutch cancer registration. Skin cancer, figures and context, current. Available from: https://www. volksgezondheidenzorg.info/onderwerp/huidkanker/ cijfers-context/huidige-situatie. Published 2019. Accessed October 20, 2019.

6. Goldenberg G, Karagiannis T, Palmer JB, et al. Incidence and prevalence of basal cell carcinoma (BCC) and locally advanced BCC (LABCC) in a large commercially insured population in the United States: A retrospective cohort study. J Am Acad Dermatol 2016;75:957-966e2.

7. Carr S, Smith C, Wernberg J. Epidemiology and risk factors of melanoma. Surg Clin North Am 2020;100:1-12.

8. Hoorens I, Vossaert K, Ongenae K, Brochez L. Is early detection of basal cell carcinoma worthwhile? Systematic review based on the WHO criteria for screening. Br J Dermatol 2016; 174:1258-1265.

9. Chen SC, Bravata DM, Weil E, Olkin I. A comparison of dermatologists' and primary care physicians' accuracy in diagnosing melanoma: a systematic review. Arch Dermatol 2001;137:1627-1634.

10. Swetter SM, Chang J, Shaub AR, Weinstock MA, Lewis ET, Asch SM. Primary Care-Based Skin Cancer Screening in a Veterans Affairs Health Care System. JAMA Dermatol 2017;153:797-801.

11. Marra E, van Rijsingen MCJ, Alkemade JAC, et al. The effect of a dermato-oncological training programme on the diagnostic skills and quality of referrals for suspicious skin lesions by general practitioners. Br J Dermatol 2021;184:538-544.

12. Ahmadi K, Prickaerts E, Smeets JGE, Joosten V, Kelleners-Smeets NWJ, Dinant GJ. Current approach of skin lesions suspected of malignancy in general practice in the Netherlands: a quantitative overview. J Eur Acad Dermatol Venereol 2018; 32:236-241.

13. Hofmans-Okkes IM, Lamberts H. The International Classification of Primary Care (ICPC): new applications in research and computer-based patient records in family practice. Fam Pract 1996;13: 294-302.

14. Luijks H, Van Boven KO, Hartman T, Uijen A, Van Weel C, Schers H. Making visible what is intuitively known by family physicians. Capturing core values of family medicine in structured data. In Press. J Am Board Fam Med 2021.

15. Oncoline. Dutch Melanoma work group. Available from: https://www.oncoline.nl/melanoom. Published 2012. Accessed November 03, 2020.

16. MedCalc Software bv. Diagnostic test evaluation calculator: MedCalc Software bv. Available from: https://www.medcalc.org/calc/diagnostic_test.php. Published 2019. Accessed November 20, 2019.

17. CBS. Population; sex, age and marital status. Available from: https://opendata.cbs.nl/statline/?dl= 308BE\#/CBS/nl/dataset/7461bev/table. Published 2020. Accessed May 10, 2021.

18. van Rijsingen MC, Hanssen SC, Groenewoud JM, van der Wilt GJ, Gerritsen MJ. Referrals by general practitioners for suspicious skin lesions: the urgency of training. Acta Derm Venereol 2014;94:138-141.

19. Leeflang MMG, Bossuyt PMM, Irwig L. Diagnostic test accuracy may vary with prevalence: implications for evidence-based diagnosis. J Clin Epidemiol 2009;62:5-12.

20. Leeflang MMG, Rutjes AWS, Reitsma JB, Hooft L, Bossuyt PMM. Variation of a test's sensitivity and specificity with disease prevalence. CMAJ 2013;185:E537-E44.

21. Verhoeven EW, Kraaimaat FW, van Weel C, et al. Skin diseases in family medicine: prevalence and health care use. Ann Fam Med 2008;6:349-54. 